\title{
Correlative In-Situ AFM \& SEM \& EDX Analysis of Nanostructured Materials
}

\author{
M. Winhold ${ }^{1}$, M. Leitner ${ }^{1}$, A. Lieb ${ }^{2}$, P. Frederix ${ }^{2}$, F. Hofbauer ${ }^{1}$, T. Strunz ${ }^{1}$, J. Sattelkov ${ }^{4}$, H. Plank ${ }^{4}$ and \\ C.H. Schwalb ${ }^{1}$ \\ 1. GETec Microscopy GmbH, Vienna, Austria. \\ 2. Nanosurf AG, Liestal, Switzerland. \\ 3. Institute for Electron Microscopy and Nanoanalysis, Graz University of Technology, Austria.
}

During the last decade the combination of different microscopic and spectroscopic methods into one instrument gained increasing importance due to the simultaneous acquisition of complementary information. Especially highly localized probing of mechanical, electrical and chemical properties on the lower nanoscale represents a key success factor for gaining new insights in the micro and nano worlds and are an indispensable task for further technological developments (e.g. new electronic- or semiconductor materials and devices).

In this work, we present a unique atomic force microscope (AFM) - the AFSEM ${ }^{\mathrm{TM}}$ - designed for seamless integration into scanning electron microscopes (SEM) or other host systems (e.g. dual beam microscopes). Its open design allows to simultaneously operate SEM and AFM inside the vacuum chamber and perform correlative in-situ AFM/SEM/EDX analysis of nanostructured materials. For the first time, this allows direct in-situ combination of complementary techniques - SEM imaging, chemical information by EDX, real 3D topography, phase information, mechanical and electrical properties by AFM. This is rendered possible by eliminating the need for optical detection and implementing novel low-noise self-sensing cantilever probes [1]. These cantilevers are equipped with a deflection sensor that directly measures the cantilever signal electrically, therefore removing the space consuming requirement for optical readout. In addition, we use a 3D nanoprinting technology (focused electron beam induced deposition, FEBID) for a variable tip modification of the cantilever structures to achieve electric conductive nanoprobes with final tip radii of $15 \mathrm{~nm}$ and below.

We present a broad variety of case studies for correlative analysis of nanostructured materials. We demonstrate results for in-situ electrical characterization of nanostructures by combining SEM and conductive AFM measurements for nanoscale dose-dependent conductivity measurements of nanogranular $\operatorname{Pt}(\mathrm{C})$ structures after focused electron beam induced processing. In addition, we use the AFM capability inside the vacuum environment of the SEM for the analysis of ultrathin structures concerning roughness, real 3D topography, and electrical properties (e.g. Black Silicon, plasma treated surfaces). We show the first interactive AFM/SEM/EDX analysis that enables the direct correlation of (sub)nanometer topography, electrical and chemical information at the exact same sample position without the need of air exposure or sample transfer (Figure 1). Finally, we present correlative AFM/SEM imaging for failure analysis of graphene films in which polymer residues lead to the degradation of electrical properties due to increased extrinsic scattering and uncontrolled doping [2]. We show how the SEM allows to quickly identify these areas while the AFM enables high resolution images of smaller areas of interest even in highly insulating regions thus gaining valuable information for device failure and performance (Figure 2). Based on the broad variety of applications regarding the characterization of semiconductor materials and devices we anticipate the AFSEM ${ }^{\mathrm{TM}}$ to be the driving characterization tool for correlative SEM/AFM analysis in the future. 


\section{References:}

[1] M Dukic, JD Adams and GE Fantner, Scientific Reports 5 (2015) 16393

[2] T Matsumae, AD Koehler, T Suga and KD Hobart, Journal of the Electromechanical Society 163 (6) E159-E161 (2016)

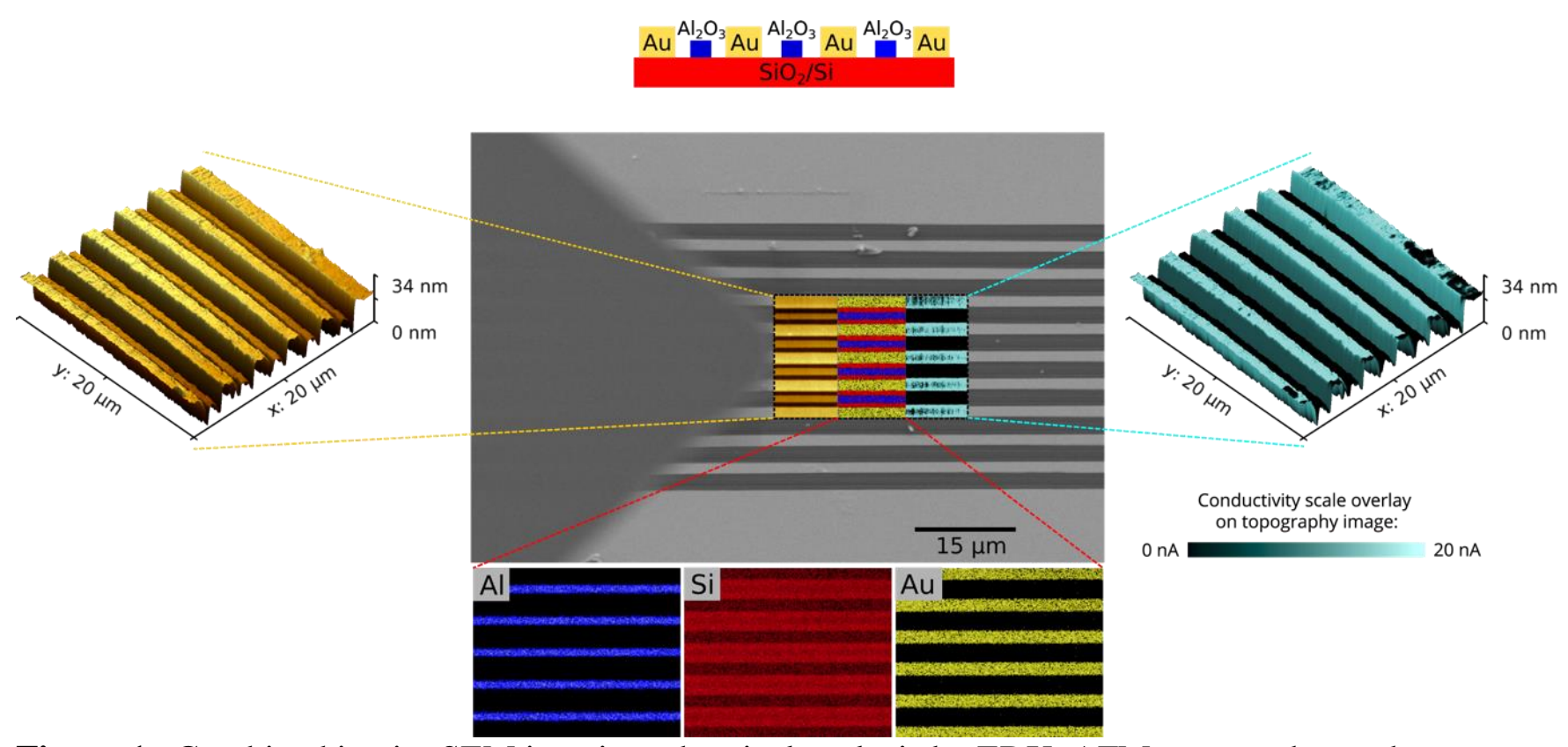

Figure 1. Combined in-situ SEM imaging, chemical analysis by EDX, AFM topography, and conductivity analysis.

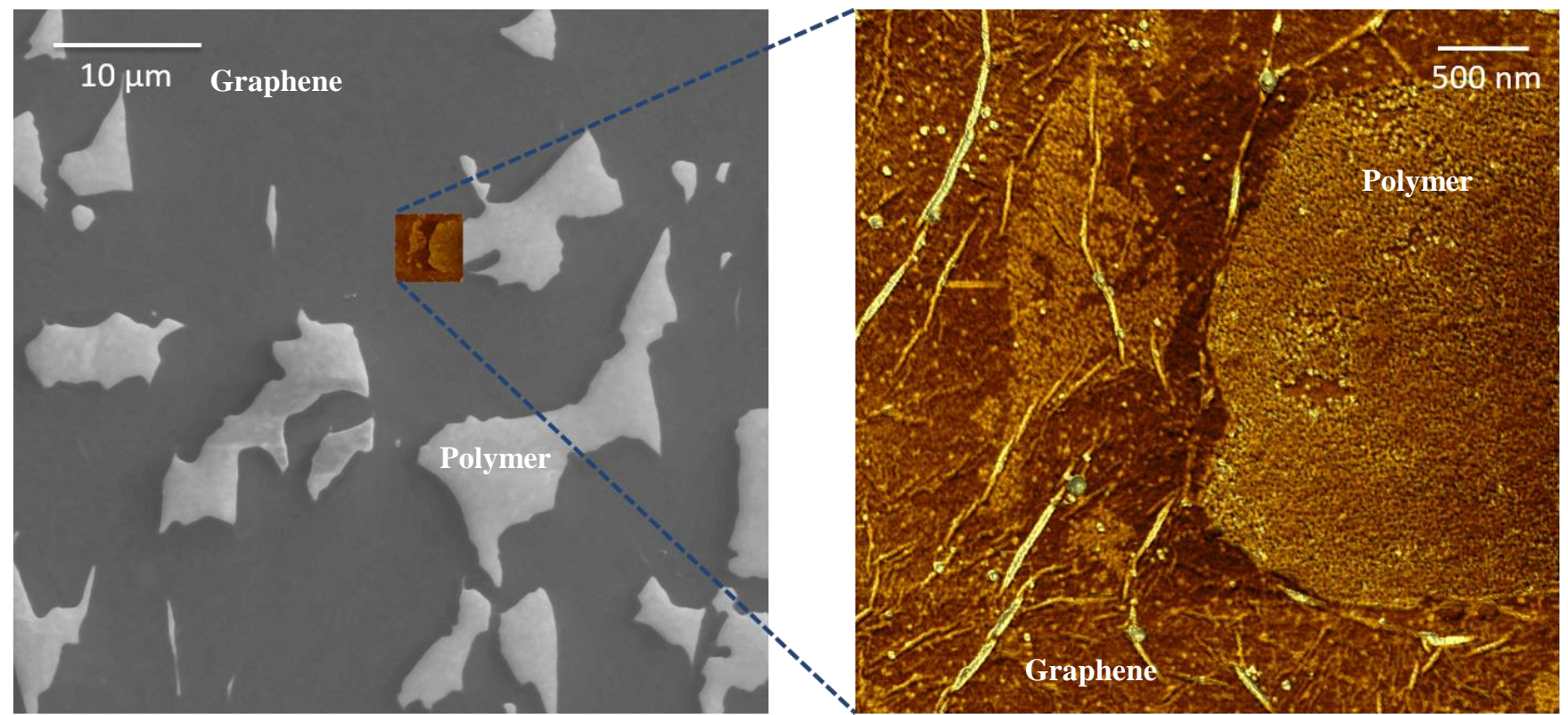

Figure 2. Correlative SEM (left) and AFM (right) in-situ analysis of graphene layers and undesired polymer residues after transfer process to an insulating substrate. 